\title{
Die Priorität von Einstellungen und Verzerrungen im Interview. Eine Methodenuntersuchung anhand der Daten der Allgemeinen Bevölkerungsumfrage 1980'
}

\author{
Dieter Hermann \\ Universität Heidelberg, Institut für Kriminologie \\ Friedrich-Ebert-Anlage 6-10, D-6900 Heidelberg
}

$\mathrm{Z}$ u $s$ a $\mathrm{m}$ m e $\mathrm{n}$ a s $\mathrm{s}$ u $\mathrm{g}$ : Die Fragestellung dieser I'ntersuchung ist, ob es Interviewer- und Befragtengruppen gibt, bei denen Interviewereffekte verstärkt auftreten. Die wesentlichen Hypothesen sind: Je geringer für den Interviewer (Befragten) die Priorität eines Fragenthemas ist, desto geringer (größer) ist der Interviewereffekt bezüglich der Fragen zu diesem Thema. Die Hypothesen werden an zwei Variablen - liberalen und leistungsorientierten Erziehungszielen - mit Hilfe der Daten der Allgemeinen Bevölkerungsumfrage der Sozialwissenschaften 1980 überprüft. Zur Uberprüfung der Hypothesen werden die Interviewer und Befragten in Subgruppen aufgeteilt, wobei sich diese Gruppen in der Einschätzung der Priorität eines Fragenthemas unterscheiden. Für jede dieser Subgruppen werden die Interviewereffekte durch Pfadanalysen mit LISREL berechnet. Die Ergebnisse: Beide Hypothesen können nicht abgelehnt werden.

\section{Theoretische Grundlagen}

In der Literatur zum Thema Interviewereffekte herrschte weitgehend Konsens daruber, daß es zwar eine große Zahl von Ad-hoc-Untersuchungen zu diesem Thema gibt, aber eine Theorie des Interviews fehit, in welche die große Zahl der Einzelbefunde integriert werden könnte (Atteslander/Kneubühler 1975: 9; Esser 1977: 253; Hoag/Allerbeck 1981: 413; Schanz/ Schmidt 1981: 9; Sudman/Bradburn 1974: 13; Kalton/Schumann 1980: 38). Ansätze, diesen Mangel zu beseitigen, stammen von Atteslander/ Kneubühler (1975) und Esser (1983).

Der Versuch von Atteslander/Kneubühler (1975) basiert auf zwei Begriffen, dem Normbegriff und dem Kostenbegriff (Atteslander/Kneubühler

1975: 12 f.). Normen sind dabei definiert als „Level of Permissiveness", d.h. sie legen fest, was erlaubt und angemessen ist oder was erwartet wird (Atteslander/Kneubühler 1975: 14). Erklärt werden soll die (verbale) Reaktion des Befragten, unabhängige Variable ist die soziale Situation Interview. Diese ist charakterisiert durch den Stimulus des Fragebogens und durch den Reiz des Interviewers und der Umgebung (Atteslander/ Kneubühler 1975: 58). Der Befragte verarbeitet

1 Für Anregungen und Kritik danke ich Günter Bollinger, Universität Mannheim, Karl-Ulrich Mayer, ZUMA, Uwe Schleth und Raymund Werle, Universität Heidelberg. diese Stimuli und Reize in einem Prozeb der Reiz- und/oder Stimulusdeutung so, daß er mit Hilfe der durch die Reize respektive Stimuli aktivierten Normen und der damit verbundenen Sanktionen aus seinem Repertoire von Reaktionsdispositionen diejenigen auswählt, die nach seiner Ansicht die geringsten Kosten verursachen (Atteslander/Kneubühler 1975: 65).

Verzerrungen im Interview werden auf die Einwirkungen von Normen zurückgeführt (Atteslander/Kneubühler 1975: 15). Der Befragte antwortet dann verzerrungsfrei, wenn der Grad der Erlaubtheit im Interview mit jenem Grad der Erlaubtheit identisch ist, der in einer alltäglichen Situation, in der sich der Befragte verhält, wirksam ist (Atteslander/Kneubühler 1975: 62).

Essers Theorie des Interviews (Esser 1983) basiert auf der kognitiven Handlungstheorie (Esser 1983: 10) und ist wesentlich differenzierter als der Versuch von Atteslander/Kneubühler (1975) ${ }^{2}$. Verzerrungen im Interview durch Befragtenreaktionen auf den Interviewer sind bei Esser (1983) durch drei Komplexe bedingt: der kulturellen SD-Tendenz ${ }^{3}$ (Tendenz, das Antwortverhalten

2 Beide Ansätze sind hier nur vereinfacht und ausschnit thaft dargestellt - ich habe mich auf Theorieelemente konzentriert, die zur Ableitung der zu prüfenden Hypothesen notwendig sind.

$3 \mathrm{SD}=$ Abkürzung von "social desirability" 
an allgemeinen, kulturellen Normen auszurichten), der situationalen SD-Tendenz (situationsspezifische Erwartungen und Befürchtungen, wie sie $2 . B$. durch den Interviewer nahegelegt werden) und der Befragtenrolle (Aufgabenorientierung des Befragten im Interview; z. B. ,wahre Antworten zu geben) (Esser 1983: $21 \mathrm{f}$.).

Der Begriff der Erwïnschtheit spielt in beiden Ansätzen eine zentrale Rolle: bei Atteslander/ Kneubühler (1975) ist er im Normbegriff enthalten und bei Esser im Begriff ,social desirability“. Die Zentralität dieses Begriffs wird auch von Scheuch (1973: 144), Sudman/Bradburn (1974:

9) und Esser (1977: 256 f.) betont. Der Befragte verzerrt also die Antworten in die Richtung, die er als erwünscht ansieht.

Die Frage ist nun: Wie werden solche „Wünsche“ vermittelt und unter welchen Bedingungen treten Interviewereffekte dieser Art auf? Die Vermittlung kann durch den Interviewer oder durch andere Personen geschehen, die während des Interviews anwesend sind. Als Vermittlungsmechanismen kommen in Frage:

- Verbale Beeinflussung (Sprechpausen, Dialekte, Befurwortung oder Ablehnung durch kurze Bemerkungen wie ,gut" oder ,Mm-hmm" (Atteslander/Kneubühler 1975: 52; Erbslöh 1973: 59 f.; Schanz/Schmidt 1981: 9).

- Nichtverbale Beeinflussung (Blickaustausch, Gestik, Mimik) (Atteslander/Kneubühler 1975: 53).

- Vermittlung über sichtbare Interviewermerkmale (Alter, Geschlecht, Kleidungsstil) (Esser 1974: 126).

Dies sind für den Befragten Indikatoren für die Einstellung des Interviewers (Esser 1974: 120) zu dem jeweiligen Fragenthema - bzw. Indikatoren der Einstellung der Personen, die eventuell zusätzlich anwesend sind. D.h., daß unter bestimmten Umständen die Einstellung des Interviewers, die Anwesenheit Dritter während des Interviews und gewisse sichtbare Merkmale des Interviewers das Befragtenverhalten beeinflussen.

Die Frage nach den Bedingungen für das Auftreten dieser Art von Interviewereffekten wurde bisher noch nicht gestellt, obwohl es schon Teilantworten darauf gibt: Nach Hoag/Allerbeck 11981: 422) wirkt sich die Anwesenheit des Ehepartners des Befragten nur dann aus, wenn Befragter und Interviewer verschiedenen Geschlechts sind. Eine weitere Bedingung ist der Frageinhalt (Sudman/Bradburn 1974: 132). Nach Erbslöh/ Wiendick (1974: 90) wirken sich sichtbare Interviewermerkmale vor allem dann auf das Antwortverhalten aus, wenn eine Beziehung zwischen dem Inhalt der Frage und dem entsprechenden Merkmal besteht. Die Wichtigkeit des Frageinhalts ist eine weitere Bedingung, die von Esser (1983: 26) genannt wird. D.h., je höher die Priorität eines Fragenthemas fur den Interviewer ist, desto intensiver werden von ihm die Mittel der verbalen und nichtverbalen Beeinflussung eingesetzt und umso größer wird der Druck auf den Befragten, Antworten zu geben, die seiner Meinung nach vom Interviewer erwünscht sind - also die vom Befragten perzipierte Einstellung des Interviewers. Es liegt nahe, die Wichtigkeit des Frageninhalts für den Befragten mitzuberücksichtigen: Je höher die Priorität des Fragenthemas für ihn ist, desto stärker wird er dem Druck des Interviewers widerstehen und nach seiner eigenen Uberzeugung antworten. Eine weitere Bedingung für das Auftreten von Interviewereffekten wird bei Esser (1983: 36) angedeutet: Die zeitliche Dauer des Interviews. Die Vermittlung der Informationen, die einer verbalen oder nichtverbalen Beeinflussung dienen, nehmen sicher Zeit in Anspruch, d.h. je länger die Befragung über einen Themenkomplex geht, desto größer ist die Wahrscheinlichkeit, daß der Befragte diese Informationen richtig versteht und sein Antwortverhalten nach der tatsächlichen Einstellung des Interviewers ausrichtet. Je größer die Anzahl der Items zu einer Einstellungsdimension ist, desto mehr paßt der Befragte sein Antwortverhalten an die Einstellung des Interviewers an.

Die Stärke des Interviewereinflusses hängt also mindestens von folgenden Bedingungen ab:

- dem Frageninhalt,

- der subjektiven Wichtigkeit des Frageninhalts

a) für den Interviewer,

b) für den Befragten,

- der Anzahl der Items zu einem Fragenthema.

Aus den eben gemachten Uberlegungen lassen sich folgende Hypothesen ableiten:

H 1: Je geringer für den Interviewer die Priorität eines Fragenthemas ist, desto geringer ist der Interviewereffekt bezüglich der Fragen zu diesem Thema.

H 2: Je geringer für den Befragten die Priorität eines Fragenthemas ist, desto größer ist 
der Interviewereffekt bezüglich der Fragen zu diesem Thema.

H 3: Je größer die Anzahl der Items zu einem Fragenthema ist, desto größer ist der Interviewereffekt.

Die dritte Hypothese kann allerdings mit dem vorhandenen Datenmaterial nur unzureichend überprüft werden.

Als Datenmaterial zur Uberprüfung dieser Hypothesen wurde die Allgemeine Bevölkerungsumfrage der Sozialwissenschaften (Allbus 1980) ${ }^{4}$ verwendet.

\section{Methodische Grundlagen}

Die Methode zur Messung von Interviewereffekten habe ich von Schanz/Schmidt (1981) übernommen. Sie definieren Interviewereffekte als diejenige Varianz, die durch Interviewermerkmale und deren Interaktion mit Befragtenmerkmalen erklärt werden kann (Schanz/Schmidt 1981: 7). Diese Explikation wird durch die Anwendung von vier multiplen Regressionsanalysen umgesetzt. Die abhängigen Variablen sind dabei Befragtenmerkmale: Liberale Erziehungsziele, Oben/unten-Einstufung, Subjektive Schichteinstufung, Zweitstimme Bundestagswahl (Sonntagsfrage), Parteisympathie und Post-Materialismus. Die unabhängigen Variablen sind:

in der 1. Regressionsanalyse: Alle substantiellen unabhängigen Variablen; das sind Befragtenmerkmale, die sie aus der Literatur entnommen haben (Schanz/Schmidt 1981: 23) und

4 Die Grundgesamtheit dieser Umfrage besteht aus allen Personen mit deutscher Staatsangehörigkeit, die in der Bundesrepublik oder West-Berlin leben und das 18. Lebensjahr vollendet haben. Aus dieser Grundgesamtheit wurden repräsentative Zufallsstichproben gezogen. Insgesamt wurden 2955 Interviews realisiert. Der Zeitpunkt der Erhebung: Januar/Februar 1980. Die Umfrage umfaßt zusätzlich eine Befragung der Interviewer. Einige Wochen vor der Durchführung der Interviews mußten die Interviewer $(N=430)$ einen Fragebogen ausfillen, der zum Teil dieselben Fragen wie die Hauptuntersuchung enthielt. Die Daten der Hauptdatei sind im Zentralarchiv für empirische Sozialforschung, Köln, archiviert. ZA-Nr. 1000, ZA-Kat. 50. Anfragen bzgl. der Interviewerdatei sind an ZUMA zu richten. Literatur $\mathbf{z u}$ methodischen Problemen des ALLBUS 1980: Mayer/ Schmidt (1983). die die jeweils abhängige Variable erklären sollen;

in der 2. Regression: Alle substantiellen Variablen, deren beta-Koeffizient größer als ein bestimmter vorgegebener Wert ist;

in der 3. Regression: $\mathrm{Zu}$ den unabhängigen $\mathrm{Va}$ riablen der 2 . Regression werden alle Interview-/Interviewermerkmale hinzugefügt, die eventuell einen Einfluß haben könnten;

in der 4. Regression: Alle substantiellen Variablen und alle Interview-/Interviewermerkmale, deren beta-Koeffizient größer als ein bestimmter vorgegebener Wert ist.

Der Interviewereffekt ist dann die Differenz der erklärten Varianzen der 2. und 4. Regression.

Bei der Anwendung von Regressionsmodellen wird unterstellt, daß die Variablen auf Intervallskalenniveau gemessen sind und die betrachteten Abhängigkeiten linear und additiv sind. Zufallige Meßfehler sollten nicht vorhanden sein, denn dadurch würden Korrelationskoeffizienten unterschätzt (Hummell/Ziegler 1976: E 113) und somit auch Determinationskoeffizienten und Interviewereffekte.

Diese Restriktionen können aber verhältnismäßig leicht überwunden werden: Das Meßfehlerproblem durch die Verwendung multipler Indikatoren in LISREL-Modellen (Weede 1977: 96) und das Problem des Skalenniveaus und der Linearität durch die Kontrolle der Ergebnisse mit einem Verfahren, das nur Ordinalskalenniveau voraussetzt: eine Pfadanalyse mit $\mathrm{r}^{*}(K)=\sin (1 / 2$. $\pi \cdot \tau)$ als Korrelationskoeffizient. Dieser Korrelationskoeffizient wurde von Devlin/Gnanadesikan und Kettenring (1975) eingeführt. Er basiert auf Kendalls Tau und unterstellt demnach nur Ordinalskalenniveau; durch die Verwendung dieses Korrelationskoeffizienten werden somit auch nichtlineare, monotone Zusammenhänge mitberücksichtigt.

Die Items zum Index „liberale Erziehungsziele“ haben eine sehr schiefe Verteilung; die Variablen sind also sicher nicht multinormalverteilt - eine Voraussetzung einer Pfadanalyse mit LISREL.

Bei Abweichungen von der Multinormalverteilung wird von Jöreskog und Sörbom (1978: 13) die Verwendung von modifizierten Korrelationskoeffizienten empfohlen. Dies ist ein weiterer Grund, eine Kontrolle der Ergebnisse mit Hilfe von $\mathbf{r}^{*}(K)$ durchzuführen. 


\section{Operationalisierungen und Untersuchungs- design}

Die Uberprüfung der Hypothesen beschränkt sich auf die Variablen liberale und leistungsorientierte Erziehungsziele ${ }^{5}$. Nur bei der ersten Variablen haben Schanz/Schmidt (1981: 55) nennenswerte Interviewereffekte gefunden - die zweite Variable wurde von ihnen nicht untersucht. Beide Variablen messen die Wichtigkeit von Erziehungszielen und können somit direkt als Prioritätsmaß verwendet werden. Die Operationalisierungen der unabhängigen Variablen übernehme ich von Schanz/Schmidt (1981: 26).

Um die Hypothese $\mathrm{Hl}$ zu testen, werden die Interviewer für jede unabhängige Variable mit Hilfe des 1. und 2. Quartils des jeweiligen Prioritätsmaßes in zwei Subgruppen unterteilt: in Interviewer, deren durchschnittliche Einstellung zu den jeweiligen Erziehungszielen kleiner oder gleich dem 1. Quartil dieser Variablen ist (Gruppe I1), und in Interviewer, deren durchschnittliche Einstellung kleiner oder gleich dem 2. Quartil (Median) dieser Variablen ist (Gruppe 12) Die Gruppe I2 enthält ca. 50\% aller Interviewer, und die Priorität der Erziehungsziele der Inter-

5 Die Fragestellung:

Liberale Erziehungsziele:

Es gibt verschiedene Eigenschaften und Fähigkeiten, die man durch Erziehung fördern kann. Wie wichtig finden Sie persönlich diese Erziehungsziele, wenn Sie an die Erziehung eines etwa zehnjährigen Kindes denken?

I1: Selbständigkeit

12: Selbstvertrauen

13: Verantwortungsbewußtsein

14: Kritikfähigkeit

15: Verständnis für andere

1: ganz unwichtig ... 7: sehr wichtig.

\section{Leistungsorientierte Erziehungsziele:}

I1: Gute Schulleistungen

I2: Fleiß.

6 Die Gruppenbildung wird an einem Beispiel deutlicher. Die Variable ,liberale Erziehungsziele der Interviewer" ist mit 5 Items $\left(I_{1} \ldots I_{5}\right)$ gemessen. Bezeichnet man mit $D_{x}$ das arithmetische Mittel dieser Items für die Person $x$, so gelten folgende Formeln für die Zusammensetzung der Gruppen 11 und 12:
$11=$
$\left\{\mid D_{x} \leqslant Q_{1}\right.$
(D) 1 ,
$12=|X| D_{x} \leqslant Q_{2}$
(D) 1 ,

wobei $Q_{1}$ das erste und $Q_{2}$ das zweite Quartil der Variablen D (durchschnittliche Liberale Erziehungsziele) ist. viewer in dieser Gruppe ist im Mittel geringer als in der Gruppe, in der alle Interviewer enthalten sind; die Gruppe II enthält ca. $25 \%$ aller Interviewer, und die Priorität der Erziehungsziele der Interviewer in dieser Gruppe ist im Mittel geringer als in der Gruppe ${ }^{2} 2^{7}$. Die Hypothese kann überprüft werden, indem man die Interviewereffekte für die einzelnen Subgruppen (I1, I2, Alle) berechnet.

Zur Prüfung von Hypothese $\mathrm{H} 2$ kann analog vorgegangen werden. Die Befragten werden mit Hilfe der Quartile in Subgruppen (B1 und B2) ${ }^{8}$ mit verschiedener Priorität der Erziehungsziele der Befragten aufgeteilt, und für jede Subgruppe wird der Interviewereffekt berechnet.

Nimmt man an, daß die Interviewereinstellung zu dem Thema Erziehungsziele die Antworten des Befragten zu diesem Thema beeinflussen, ist die Gruppenbildung bei den Befragten zum Teil auf einen Interviewereffekt zurückzuführen. Um die Auswirkungen dieses Interviewereffekts auf die Untersuchungsergebnisse tendenziell abschät. zen zu können, wird die Gruppenbildung bei den Befragten zusätzlich so durchgeführt, daß die durchschnittliche Befragteneinstellung aus Items berechnet wird, aus denen der Einfluß der Interviewereinstellung herauspartialisiert wurde. Das Vorgehen wird an einem Beispiel deutlich: Der (lineare) Einfluß der Interviewereinstellung zu liberalen Erziehungszielen auf die Befragteneinstellung zu diesem Thema kann über eine Regressionsanalyse geschätzt werden. Die Regressionsgleichungen:

$\mathbf{J}_{\mathrm{i}}=\mathrm{a}_{\mathbf{i}} \cdot \mathrm{I}_{\mathbf{i}}+\mathrm{b}_{\mathrm{i}}+\mathbf{R}_{\mathrm{i}} \quad(\mathrm{i}=1, \ldots, 5)$

7 Die naheliegende Art der Gruppierung - die Interviewer in disjunkte Gruppen mit hoher und niederer Priorität aufzuteilen - kann hier nicht durchgeführt werden, denn die Items haben eine sehr schiefe Verteilung. Dadurch werden die Varianz dieser Items in der Gruppe mit hoher Priorität sehr klein und die Validität der Messungen gering.

8 Zur Verdeutlichung ein Beispiel.

Die Variable „liberale Erziehungsziele des Befragten“" ist mit 5 Items $\left(\mathrm{J}_{1}, \ldots \mathrm{J}_{5}\right)$ gemessen Bezeichnet man mit $\mathbf{M}_{\mathbf{X}}$ das arithmetische Mittel dieser Items für die Person $x$, so gelten folgende Formeln für die Zusammensetzung der Befragtengruppen:

$B 1=|x| M_{X} \leqslant Q_{1}(M) \mid$,

$B 2=|x| M_{x} \leqslant Q_{2}(M) \mid$,

wobei $M$ der Durchschnitt von $J_{1}, \ldots J_{5}$ ist. 
$\left(\mathrm{J}_{\mathrm{i}}\right.$ : Befragteneinstellung, $\mathrm{I}_{\mathrm{i}}$ : Einstellung des Interviewers, $\mathrm{a}_{\mathrm{i}}$ : unstandardisierter Regressionskoeffizient, $\mathbf{R}_{\mathbf{i}}$ : Residuum).

Die Variable $\mathbf{R}_{\mathbf{i}}$ mißt die Einstellung des Befragten $z u$, liberalen Erziehungszielen', die von der Einstellung des Interviewers unabhängig ist und somit mißt das arithmetische Mittel dieser $5 \mathrm{Va}$ riablen $R_{\mathbf{i}}$ die durchschnittliche Befragteneinstellung, aus der die Interviewereinstellung herauspartialisiert wurde. Die Quartile dieser Variablen werden verwendet, um die Befragten in Subgruppen $(\overline{B 1}, \overline{B 2})^{9}$ aufzuteilen.

\section{Damit erhält man folgendes Design:}

\section{Variablenauswahl:}

Für jede der unabhängigen Variablen liberale und leistungsorientierte Erziehungsziele werden vier multiple Regressionen durchgefuhrt; Modell 2 enthält alle wesentlichen ${ }^{10}$ substantiellen Variablen und Modell 4 zusätzlich alle wesentlichen Interview-/Interviewermerkmale als unabhängige Variable.

\section{Schätzung der Parameter:}

Die Parameter der Modelle 2 und 4 werden mit LISREL berechnet, sowohl mit Pearsons' $r$ als auch mit $\mathrm{r}^{*}(\mathrm{~K})$ als Korrelationskoeffizienten.

Die Parameter der beiden Modelle werden grundsätzlich für folgende Populationen berechnet:

a) für alle Interviewer und Befragten,

b) für Interviewersubgruppen (I1, I2),

c) für Befragtensubgruppen (B1, B2),

d) für Befragtensubgruppen, wobei bei der Gruppenbildung der Einfluß der Interviewer berücksichtigt wird $(\overline{\mathrm{B} 1}, \overline{\mathrm{B} 2})$.

9 Ist $\mathbf{R}_{\mathbf{X}}$ das arithmetische Mittel der 5 Variablen $\mathbf{R}_{\mathbf{i}}$ $(i=1, \ldots, 5)$ der Person $x$, so sind die Gruppen folgendermaßen definiert:

$\left.\overline{B T}=|x| R_{x} \leqslant Q_{1}(R)\right\}$, $\overline{B 2}=|x| R_{X} \leqslant Q_{2}(R) \mid$,

10 ,Wesentlich' heißt, daß die Pfadkoeffizienten zu diesen Variablen größer als ein bestimmter, vorgegebener Wert sind.

\section{Engebnisse}

\subsection{Liberale Erziehungsziele}

Diese Variable wurde von Schanz/Schmidt (1981) schon untersucht, und es ist in ihrer Studie die einzige Variable, bei der ein Interviewereffekt feststellbar ist. Durch Interviewermerkmale können zusätzlich $8 \%$ mehr an Varianz erklärt werden (Schanz/Schmidt 1981: 57). Die Ergebnisse der vier multiplen Regressionen stehen in der nachfolgenden Tabelle 1 (aus Schanz/Schmidt 1981: 46).

Dieses Verfahren wird hier als Ausleseverfahren für die wesentlichen unabhängigen Variablen angesehen; man kann also die Analyse auf die Befragtenmerkmale: Alter, Haushaltseinkommen, Schichteinstufung, Post-Materialismus-Score, und

TABELLE 1 Liberale Erziehungsziele

Die Parameterschätzungen sind standardisierte multiple Regressionskoeffizienten

Nr. der Regressionsanalyse

\begin{tabular}{lllll} 
Variable & 1 & 2 & 3 & 4 \\
\hline
\end{tabular}

Befragten-Merkmale:

Alter

Geschlecht

$-.053-.056-.047-.049$

Schulbildung

.039

Berufsprestige

Einkommen

Haushaltseinkommen

Oben/unten-Skala

Schichteinstufung

Post-Materialismus-Score

Konfession

$-.013$

$-.012$

.031

$-.053$

$-.018$

.076

$-.040-.029-.023$

Kirchgangshäufigkeit

$-.015$

$\begin{array}{lll}.064 & .071 & .071\end{array}$

$\begin{array}{lll}.097 & .085 & .082\end{array}$

Interviewer-Merkmale:

Alter

Geschlecht

$-.043$

Schulbildung

berufliche Stellung

Konfession

Kirchgangshäufigkeit

Erziehungsziele

Anzahl Interviews 1979

Zeitverfügbarkeit

$-.025$

Interview-Situation:

Dritte während des Inter- 


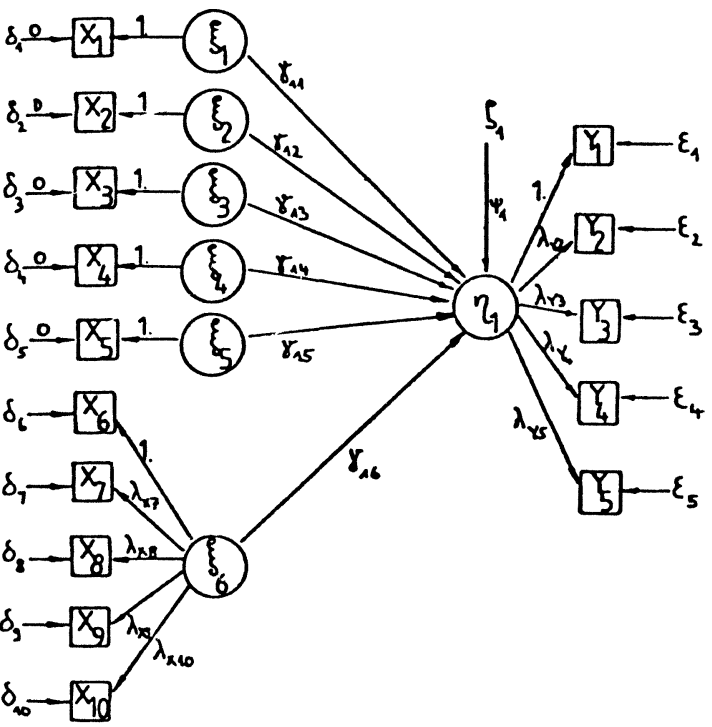

Legende:

Interviewermerkmale

Befragtenmerkmale

$\xi_{5}:$

Berufliche Stellung

$\xi_{1}:$ Alter

Liberale Erziehungsziele
- Befragtengruppen mit unterschiedlicher Einschätzung der Priorität von liberalen Erziehungszielen - sowohl mit als auch ohne Berücksichtigung von Interviewereffekten bei der Gruppenbildung (B1, B2, $\overline{\mathrm{B} 1}, \overline{\mathrm{B} 2}$ ),

mit Pearsons' $r$ und $r^{*}(K)$ als Korrelationskoeffizienten. Die Ergebnisse stehen in den nachfolgenden Tabellen 2 und 3.

Die Interpretation dieser Ergebıisse ist methodisch und inhaltlich interessant:

\section{Die methodische Ebene:}

Betrachtet man alle Befragten, so kann man durch Interviewermerkmale $12,8 \%$ mehr an $\mathrm{Va}$ rianz erklären (im Vergleich dazu: $8 \%$ sind es bei Schanz/Schmidt (1981)). Die Nichtberücksichtigung zufälliger Meßfehler führt also zu einer Unterschätzung des Interviewereffekts, ebenso die Nichtberücksichtigung des Meßniveaus und eventuell vorhandener, nichtlinearer, aber monotoner Zusammenhänge, denn als Schätzung des Interviewereffekts auf der Basis von $r^{*}(\mathrm{~K})$ erhält man für die gleiche Population einen $\mathrm{Zu}$ wachs der erklärten Varianz durch Interviewermerkmale von $16,8 \%$.

\section{Die inhaltliche Ebene:}

Je geringer die Priorität des Fragenthemas für den Interviewer ist, desto geringer ist der Interviewereffekt, und je geringer die Priorität des Fragenthemas für den Befragten ist, desto gröBer ist der Interviewereffekt. Die Hypothesen H1 und H2 können somit nicht abgelehnt werden. Verantwortlich für den relativ großen Interviewereffekt ist der Pfadkoeffizient zwischen den Erziehungszielen des Interviewers und den Erziehungszielen des Befragten. Die Befragten orientieren sich also vorwiegend an der Einstellung des Interviewers zu diesem Thema. Eine Ausnahme bildet die Gruppe I1. Nur in dieser ist der direkte Effekt, der vom Beruf des Interviewers ausgeht, größer als der direkte Effekt, der von der Einstellung des Interviewers ausgeht. Dies kann folgendermaßen interpretiert werden: Bei sehr geringer Priorität des Fragenthemas für den Interviewer ,,verzichtet" der Interviewer weitgehend auf verbale und nichtverbale Einflußmechanismen, die dem Befragten seine Einstellung vermitteln. Der Befragte kann
11 Dabei bedeuten:

$\xi: \quad$ latente exogene,

$\eta$ : latente endogene Variablen

$\mathrm{X}, \mathrm{Y}$ : Indikatoren zu den latenten Variablen

5: Residuen zu den latenten endogenen Variablen

$\epsilon, \delta$ : Meßfehler der Indikatoren. 
TABELLE 2 Liberale Erziehungsziele Die Parameterschätzungen sind standardisierte Werte. Korrelationsmatrix: Pearsons' $r$ bzw, $r^{*}(K)$

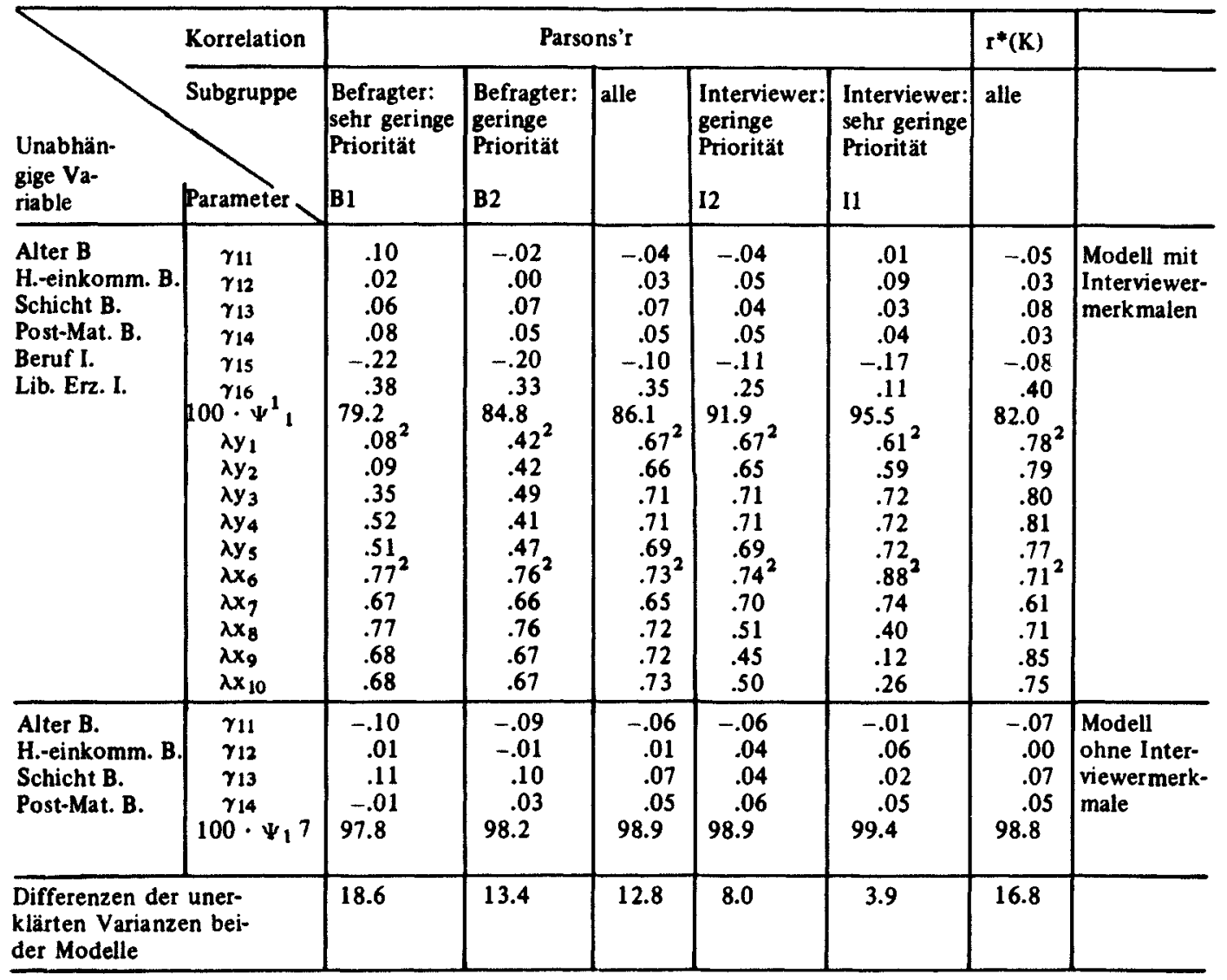

(1): Der Parameter ist interpretierbar als unerklärte Varianz (in \%).

(2): Vorgegebene Werte

sich jetzt nur noch an sichtbaren ${ }^{12}$ Merkmalen des Interviewers orientieren - sie sind der einzige Anhaltspunkt, die ,erwünschte“ Antwort zu erraten. Trifft also ein Befragter auf einen Interviewer, dem das Fragenthema wichtig ist, orientiert sich der Befragte vorwiegend an der Einstellung des Interviewers zu diesem Thema; trifft ein Befragter auf einen Interviewer, dem das Fragenthema sehr unwichtig ist, orientiert er sich an sichtbaren Merkmalen des Interviewers, die er als Indikatoren für die Einstellung des Interviewers interpretiert.

12 Die berufliche Stellung (Arbeiter/Angestellte und Beamte/Akademiker) ist natürlich nur indirekt sichtbar.
Um die Auswirkungen des Interviewereffekts auf die Gruppenbildung bei den Befragten B1 und B2 abschätzen zu können, kann man die Gruppenbildung so vornehmen, daß der lineare Effekt der Einstellung des Interviewers auf die Einstellung des Befragten herauspartialisiert wird und die Gruppenbildung mit den ,bereinigten“" Antworten der Befragten durchgeführt wird. Die Tabelle 3 enthält einen Vergleich beider Arten der Bildung von Befragtengruppen.

Vergleicht man die „Differenzen der unerklärten Varianzen beider Modelle" in dieser Tabelle, sieht man, daB die Interviewereffekte unterschätzt werden, wenn bei der Bildung von Befragtengruppen der EinfluB der Interviewer unberücksichtigt bleibt. Die Interviewereffekte 
TABELLE 3 Liberale Erziehungsziele: Auswirkung von Interviewereffekten auf die Bildung von Befragtengruppen. Die Parameterschätzungen sind standardisierte Werte. Korrelationsmatrix: Pearsons' $r$

\begin{tabular}{|c|c|c|c|c|c|}
\hline \multirow[b]{2}{*}{$\begin{array}{l}\text { Unabhängige } \\
\text { Variable; Para- } \\
\text { meter }\end{array}$} & \multicolumn{2}{|c|}{ Befragtenverhalten } & \multicolumn{2}{|c|}{$\begin{array}{l}\text { Befragtenverhalten unter } \\
\text { Berücksichtigung von Inter- } \\
\text { viewereffekten }\end{array}$} & \\
\hline & $\begin{array}{c}\text { sehr gering } \\
\text { B1 }\end{array}$ & $\begin{array}{l}\text { gering } \\
\text { B2 }\end{array}$ & $\begin{array}{c}\text { sehr gering } \\
\overline{\mathrm{B} 1}\end{array}$ & $\begin{array}{l}\text { gering } \\
\overline{\mathrm{B} 2}\end{array}$ & \\
\hline $\begin{array}{l}\text { Alter B. } \\
\text { H. einkomm. B. } \\
\text { Schicht B. } \\
\text { Post-Mat. B. } \\
\text { Beruf I. } \\
\text { Lib. Erz. I. } \\
\text { Unerkl. Varianz (\%) }\end{array}$ & $\begin{array}{r}.10 \\
.02 \\
.06 \\
.08 \\
-.22 \\
.38 \\
79.2\end{array}$ & $\begin{array}{r}-.02 \\
.00 \\
.07 \\
.05 \\
-.20 \\
.33 \\
84.8\end{array}$ & $\begin{array}{r}.06 \\
.02 \\
.09 \\
.01 \\
-.22 \\
.58 \\
60.6\end{array}$ & $\begin{array}{r}.02 \\
.02 \\
.06 \\
.08 \\
-.16 \\
.49 \\
73.0\end{array}$ & $\begin{array}{l}\text { Modell mit Inter- } \\
\text { viewermerkmalen }\end{array}$ \\
\hline $\begin{array}{l}\text { Alter B. } \\
\text { H. einkomm. B. } \\
\text { Schicht B. } \\
\text { Post-Mat. B. } \\
\text { Unerkl. Varianz (\%) }\end{array}$ & $\begin{array}{r}-.10 \\
.01 \\
.11 \\
-.01 \\
97.8\end{array}$ & $\begin{array}{r}-.09 \\
-.01 \\
.10 \\
.03 \\
98.2\end{array}$ & $\begin{array}{r}.06 \\
-.02 \\
.07 \\
.03 \\
99.1\end{array}$ & $\begin{array}{r}.01 \\
-.01 \\
.07 \\
.10 \\
98.6\end{array}$ & $\begin{array}{l}\text { Modell ohne } \\
\text { Interviewermerk- } \\
\text { male }\end{array}$ \\
\hline $\begin{array}{l}\text { Diffcrenz der unerklärten } \\
\text { Varianzen beider Modelle }\end{array}$ & 18.6 & 13.4 & 38.5 & 25.6 & \\
\hline
\end{tabular}

können in bestimmten Befragtengruppen erstaunlich hohe Werte annehmen: Durch Interviewermerkmale kann in der Gruppe $\overline{\mathrm{B}} 1$ zusätz. lich ca. 38\% der Varianz der Befragteneinstellung erklärt werden. Dieser Wert wird zudem noch durch die Verwendung von Pearsons' $r$ als Korrelationskoeffizient unterschätzt.

\subsection{Leistungsorientierte Erziehungsziele}

Tendenziell erhält man dieselben Ergebnisse wie bei der Variablen „liberale Erziehungsziele“. Die Ergebnisse sollen deshalb gerafft dargestellt werden ${ }^{13}$. Die Ergebnisse der vier multiplen Regressionen (Ausleseverfahren für die wesentlichen unabhängigen Variablen) stehen in der Tabelle 4.

Die LISREL-Modelle für die wesentlichen unabhängigen Variablen werden für die gleichen Interviewer- und Befragtengruppen geschätzt wie bei der Variable ,liberale Erziehungsziele“. Die Ergebnisse stehen in Tabelle 5.

13 Interessenten sende ich weitere Informationen gerne zu.
Bei der Interpretation der Werte in Tabelle 5 muß man allerdings berücksichtigen, daß in den Gruppen B1, $\overline{B 1}$ und 11 die Faktorladungen der Items zu den leistungsorientierten Erziehungszielen des Interviewers bzw. des Befragten stark variieren $^{14}$ - die Validität der Messung dieser Variablen ist nicht mehr gesichert. So wird erklärbar, daß der Interviewereffekt in der Befragtengruppe mit geringer Priorität größer ist als in der Befragtengruppe mit sehr geringer Priorität. Das Niveau des Interviewereffekts ist bei der Variable "leistungsorientierte Erziehungsziele" niedriger als bei der Variable ,liberale Erziehungsziele". Dies kann zwei Gründe haben: Der modifizierte Frageninhalt oder die geringere $\mathbf{A n}$ zahl der Items zu dieser Dimension ${ }^{15}$ (Hypothese H3).

14 Dies trifft auch für die Gruppe B1 bei der Variablen "liberale Erziehungsziele" zu.

15 Die Variable „Liberale Erziehungsziele“ ist mit 5 Items gemessen, die andere mit 2 Items. 
TABELLE 4 Leistungsorientierte Erziehungsziele. Die Parameterschätzungen sind standardisierte, multiple Regressionskoeffizienten

Nr. der Regressionsanalyse

\begin{tabular}{lrrrr} 
Variable & 1 & 2 & 3 & 4 \\
\hline Befragten-Merkmale: & & & & \\
Alter & .17 & .17 & .16 & .17 \\
Geschlecht & -.01 & & & \\
Schulbildung & -.22 & -.22 & -.19 & -.19 \\
Berufsprestige & .03 & & & \\
Einkommen & -.02 & & & \\
Haushaltseinkommen & .02 & & & \\
oben/unten Skala & .05 & & & \\
Schichteinstufung & .00 & & & \\
Post-Materialismus Score & -.11 & -.12 & -.11 & -.11 \\
Konfession & -.02 & & & \\
Kirchgangshäufigkeit & -.02 & & &
\end{tabular}

Interviewer-Merkmale:

$\begin{array}{lll}\text { Alter } & .03 & \\ \text { Geschlecht } & .05^{\mathrm{x}} & \\ \text { Schulbildung } & -.07 & -.07 \\ \text { berufliche Stellung } & .02 & \\ \text { Konfession } & .02 & \\ \text { Kirchgangshäufigkeit } & -.00 & \\ \text { Erziehungsziele } & .16 & .17 \\ \text { Anzahl Interviews 1979 } & .01 & \\ \text { Zeitverfügbarkeit } & -.02 & \end{array}$

Interviewer-Situation:

Dritte während des Interviews anwesend $-.06-.06$
Gruppe mit extrem hohen Interviewereffekten

\begin{tabular}{|c|c|c|c|}
\hline $\begin{array}{l}\text { unabhängige } \\
\text { Variable }\end{array}$ & $\mathbf{p}$ & $\begin{array}{l}\text { wahre Befragten- } \\
\text { einstellung } \\
\text { gemessene } \\
\text { Befragten- } 1 . \\
\text { einstellung }\end{array}$ & $\begin{array}{l}\text { wahre } \\
\text { Intervie- } \\
\text { werein- } \\
\text { stellung }\end{array}$ \\
\hline $\begin{array}{l}\text { Effekt von der } \\
\text { messenen Befr }\end{array}$ & & $\begin{array}{l}\text { Igigen Variablen } z \\
\text { tellung: } p .0=0\end{array}$ & ge- \\
\hline
\end{tabular}

Gruppe ohne Interviewereffekte:

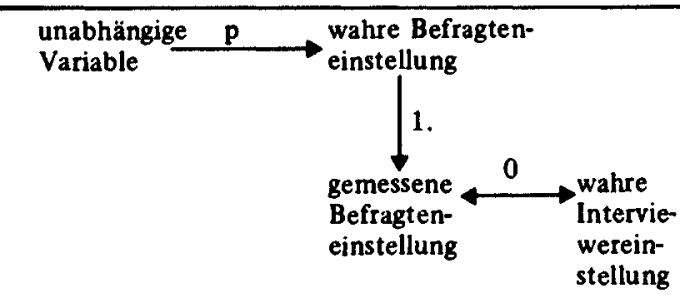

Effekt von der unabhängigen Variablen zur gemessenen Befragteneinstellung: p.1 = p.

ABBILDUNG 1 Vergleich von Befragtengruppen mit unterschiedlich starken Interviewereffekten

\section{Zusammenfassung, Auswirkungen und Schlusfolgerungen}

Sowohl die Priorität der Einstellung des Interveiwers als auch die des Befragten hat sich als wichtige Randbedingung für das Auftreten von Interviewereffekten erwiesen. Je geringer die Priorität des Fragenthemas für den Interviewer ist, desto geringer ist der Interviewereffekt und je geringer die Priorität des Fragenthemas für den Befragten ist, desto größer ist der Interviewereffekt. Tabelle 6 zeigt eine Ubersicht über die Stärke der Interviewereffekte bei beiden untersuchten Variablen.

Je höher die Priorität des Fragenthemas beim Interviewer ist, desto stärker orientiert sich der
Befragte an der Einstellung des Interviewers. Ist die Priorität beim Interviewer sehr gering - fehlen also weitgehend die verbalen und nichtverbalen Informationen, die dem Befragten als Indikatoren für die Einstellung des Interviewers dienen -, orientiert sich der Befragte weitgehend an sichtbaren Interviewermerkmalen, die er ebenfalls als Indikatoren für die Interviewereinstellung interpretiert - auf jeden Fall wird vom Befragten seine Antwort in die Richtung der perzipierten Interviewermeinung verzerrt.

Es gibt also Interviewer- und Befragtengruppen, in denen Interviewereffekte verstärkt bzw. abgeschwächt auftreten. Die Frage ist nun, welche Auswirkungen solche gruppenspezifisch auftretende Interviewereffekte auf die Schätzung von 
TABELLE 5 Leistungsorienticrte Erziehungsziele. Die Parameterschätzungen sind standardisierte Werte. Korrelationsmatrix: Pearsons'r $\mathrm{r}^{*}(\mathrm{~K})$

\begin{tabular}{|c|c|c|c|c|c|c|c|c|c|}
\hline \multirow{3}{*}{$\begin{array}{l}\begin{array}{l}\text { Korrelation } \\
\text { Kriterium } \\
\text { der Grup- } \\
\text { penbildung }\end{array} \\
\text { Unabhängige } \\
\text { Variable: } \\
\text { Parameter } \\
\text { Sub- } \\
\text { Priorio } \\
\text { tät }\end{array}$} & \multicolumn{7}{|c|}{ Pearsons' r } & $r^{*}(K)$ & \\
\hline & \multicolumn{5}{|c|}{ Befragtenverhalten } & \multicolumn{2}{|c|}{\begin{tabular}{|l}
$\begin{array}{l}\text { Befragtenverhalten } \\
\text { unter Berücksich- } \\
\text { tigung von Inter- } \\
\text { viewerverhalten }\end{array}$ \\
Befragte
\end{tabular}} & $\begin{array}{l}\text { Befragten- } \\
\text { verhalten }\end{array}$ & \\
\hline & $\begin{array}{c}\text { I } \\
\text { sehr ge } \\
\text { II }\end{array}$ & $\begin{array}{l}\text { wer } \\
\text { gering } \\
\text { I2 }\end{array}$ & alle & $\begin{array}{r}\mathrm{Be} \\
\text { geri } \\
\mathbf{B 2}\end{array}$ & $\begin{array}{l}\text { gete } \\
\text { sehr gerin } \\
\text { B1 }\end{array}$ & $\frac{\text { gerin }}{\mathrm{B} 2}$ & $\begin{array}{l}\text { fragte } \\
\text { sehr gering } \\
\frac{\mathrm{B} 1}{}\end{array}$ & alle & \\
\hline $\begin{array}{l}\text { Alter B. } \\
\text { Schulbildung B. } \\
\text { Post-Mat. B. } \\
\text { Anwesenh. Dritt. } \\
\text { Schulbildung I. } \\
\text { Leistungsor. Erz. I. } \\
\text { Unerkl. Varianz } \\
\text { (\%) }\end{array}$ & $\begin{array}{r}.25 \\
-.23 \\
-.23 \\
-.11 \\
-.01 \\
.02 \\
81.8\end{array}$ & $\begin{array}{r}.23 \\
-.24 \\
-.17 \\
-.09 \\
-.05 \\
.13 \\
82.5\end{array}$ & $\begin{array}{c}.21 \\
-.22 \\
-.16 \\
-.09 \\
-.06 \\
.22 \\
81.5\end{array}$ & $\begin{array}{r}.28 \\
-.16 \\
-.17 \\
-.18 \\
-.07 \\
.30 \\
72.2\end{array}$ & $\begin{array}{r}.23 \\
-.15 \\
-.11 \\
-.17 \\
-.04 \\
.33 \\
76.7\end{array}$ & $\begin{array}{r}.21 \\
-.14 \\
-.15 \\
-.15 \\
-.04 \\
.64 \\
50.3\end{array}$ & $\begin{array}{r}.14 \\
-.06 \\
-.13 \\
-.17 \\
-.03 \\
.50 \\
67.1\end{array}$ & $\begin{array}{r}.19 \\
-.24 \\
-.18 \\
-.09 \\
-.04 \\
.21 \\
81.0\end{array}$ & $\begin{array}{l}\text { Modell } \\
\text { mit } \\
\text { Inter- } \\
\text { viewer- } \\
\text { merk- } \\
\text { malen }\end{array}$ \\
\hline $\begin{array}{l}\text { Alter B. } \\
\text { Schulbildung B. } \\
\text { Post-Mat. } \\
\quad \text { Unerkl. Varianz } \\
\text { \% }\end{array}$ & $\begin{array}{r}.25 \\
-.24 \\
-.23 \\
82.7\end{array}$ & $\begin{array}{r}.23 \\
-.27 \\
-.18 \\
84.3\end{array}$ & $\begin{array}{r}.21 \\
-.25 \\
-.17 \\
86.4\end{array}$ & $\begin{array}{l}.29 \\
-.22 \\
-.22 \\
82.3\end{array}$ & $\begin{array}{r}.28 \\
-.21 \\
-.15 \\
85.9\end{array}$ & $\begin{array}{r}.25 \\
-.22 \\
-.21 \\
84.9\end{array}$ & $\begin{array}{r}.25 \\
-.15 \\
-.17 \\
88.4\end{array}$ & $\begin{array}{r}.19 \\
-.29 \\
-.18 \\
84.2\end{array}$ & $\begin{array}{l}\text { Modell } \\
\text { ohne } \\
\text { Inter- } \\
\text { viewer- } \\
\text { merk- } \\
\text { malen }\end{array}$ \\
\hline $\begin{array}{l}\text { Differenzen der } \\
\text { unerklärten Varian- } \\
\text { zen beider Modelle }\end{array}$ & .09 & 1.8 & 4.9 & 10.1 & 9.2 & 34.6 & 21.3 & 3.2 & \\
\hline
\end{tabular}

TABELLE 6 Ubersicht über die Stärke von Interviewereffekten. Die Parameterschätzungen sind Prozentpunktdifferenzen

\begin{tabular}{|l|lllll}
\hline $\begin{array}{l}\text { Sub- } \\
\text { gruppe }\end{array}$ & \multicolumn{2}{l}{$\begin{array}{l}\text { Priorität } \\
\text { beim Interviewer }\end{array}$} & $\begin{array}{l}\text { Priorität } \\
\text { beim }\end{array}$ \\
\cline { 2 - 7 } Variable & I1 & 12 & alle & B2 & B1 \\
\hline Liberale Erz. & 3.9 & 7.0 & 12.8 & 13.8 & 18.8 \\
Leistungs. Erz. & 0.9 & 1.8 & 4.8 & 10.1 & 9.2 \\
\hline
\end{tabular}

Pfad- oder Korrelationskoeffizienten haben. Die Auswirkungen werden deutlich, wenn man in einem Gedankenexperiment den Extremfall dieses Fehlertyps betrachtet: Eine Befragtengruppe antwortet unverzerrt, während die andere völlig die Meinung des Interviewers übernimmt. Nimmt man an, daß die Interviewer- und wahre Befragteneinstellung unabhängig voneinander sind, so bedeutet das, daß in der 2 . Gruppe zwischen wahrer und geäußerter Befragteneinstellung eine Korrelation von Null existiert. Somit haben alle Variablen, die einen Einfluß auf die wahre Befragteneinstellung haben, keinen Effekt auf die gemessene Befragteneinstellung. Deutlich wird dies in der Abbildung 1.

Aus der Ubersicht wird deutlich, daß der Effekt von der unabhängigen Variablen zur gemessenen Befragteneinstellung deflationiert wird, wenn beide Gruppen zusammengefaßt werden. Gruppenspezifisch wirkende Interviewereffekte bewirken also eine Unterschätzung des Betrags der Pfad- und somit auch der Korrelationskoeffizienten. 
Falls die Ergebnisse dieser Untersuchung durch weitere Umfragen bestätigt werden sollten, bedarf es einer Revision des Meßinstruments Interview. (Bei weiteren Untersuchungen zu diesem Themenbereich wäre es allerdings sinnvoll, die Einstellung des Interviewers und des Befragten und die Prioritäten dieser Einstellungen getrennt abzufragen). Hoag/Allerbeck (1981: 425) schlagen als Revisionsmöglichkeit eine Integration der Meßvorschriften in die inhaltliche Theorie vor. Dies hätte zur Folge, daß jede inhaltliche Theorie für jedes Meßinstrument (schriftliche Befragung, Interview, Telefoninterview, . . . ) modifiziert werden müßte. Sinnvoller erscheinen mir folgende Versuche:

1. Die Einstellungen der Interviewer und die Priorität dieser Einstellungen für Interviewer und Befragten werden bei jeder Befragung miterfaßt und in den Analysen kontrolliert. Kausalanalysen könnten für Interviewer, denen das Fragenthema wichtig ist, und Interviewer, denen es unwichtig ist, getrennt durchgefuhrt werden ${ }^{16}$.

\section{Durch eine stärkere „Uniformierung“ des In-} terviews könnten die Mechanismen, die dem Befragten die Einstellung des Interviewers vermitteln, unterbunden werden. Damit wäre die Quelle für die verstärkten Interviewereffekte zum Teil beseitigt.

\section{Literatur:}

Atteslander, P./Kneubühler, H.U., 1975: Verzerrungen im Interview. $\mathrm{Zu}$ einer Fehlertheorie der Befragung. Opladen: Westd. Verlag.

Devlin, S.J./Gnanadesikan, R./Kettenring, J.R., 1975: Robust Estimation and Outlier Detection with Correlation Coefficients. Biometrika 62: $531-545$.

16 Der Vorschlag stammt von Karl Ulrich Mayer, ZUMA.
Erbslöh, E., 1973: Die unkontrollierte Einflußnahme des Interviewers im Forschungsinterview. S. 3-67 in: R. König/R. Mayntz (Hrsg.), Studien zum Interview. Meisenheim am Glan: Hain.

Erbslöh, E./Wiendieck, G., 1974: Der Interviewer. S. 83-106 in: J.V. Koolwijk/M. Wieken-Mayser (Hrsg.), Techniken empirischer Sozialforschung. Band 4. München/Wien: Oldenbourg.

Esser, H., 1974: Der Befragte. S. 107-145 in: J.V. Koolwijk/M. Wieken-Mayser (Hrsg.), Techniken empirischer Sozialforschung. Band 4. München/Wien: Oldenbourg.

Esser, H., 1975: Soziale Regelmäßigkeiten des Befragtenverhaltens. Meisenheim am Glan: Hain.

Esser, H., 1977: Response Set - Methodische Problematik und soziologische Interpretation. Zeitschrift für Soziologie 6: 253-263.

Esser, H., 1983: Determinanten des Interviewer- und Befragtenverhaltens: Probleme der theoretischen Erklärung und empirischen Untersuchung von Interviewereffekten. Erscheint in: K.U. Mayer/P. Schmidt (Hrsg.), Allgemeine Bevölkerungsumfrage der Sozialwissenschaften - Beiträge zu methodischen Problemen des ALLBUS 1980. Frankfurt: Campus.

Hoag, W.J./Allerbeck, K.R., 1981: Interviewer- und Situationseffekte in Umfragen: Eine log-lineare Analyse. Zeitschrift für Soziologie 10: 413-426.

Hummell, H.J./Ziegler, R., 1976: Zur Verwendung linearer Modelle bei der Kausalanalyse nicht-experimenteller Daten. S. E5-E137 in: H.J. Hummell/R. Ziegler (Hrsg.), Korrelation und Kausalität. Band 1. Stuttgart: Enke.

Jöreskog, K.G./Sörbom, D., 1978: LISREL IV. Uppsala.

Kalton, G./Schumann, H., 1980: The Effect of the Question in Survey Responses: A Review. Unveröffentlichtes Manuskript. ASA, Houston, Texas.

König, R., 1974: Das Interview - Formen, Technik, Auswertung. Köln: Kiepenheuer \& Witsch.

Kriz, J., 1981 : Methodenkritik empirischer Sozialforschung. Stuttgart: Teubner.

Mayer, K.U./Schmidt, P., (Hrsg.), 1983: Allgemeine Bevölkerungsumfrage der Sozialwissenschaften Beiträge zu methodischen Problemen des Allbus 1980. Frankfurt: Campus.

Reschka, W., 1973: Einige Aspekte der verbalen Interaktion im Interview. S. 143-185 in: R. König/R. Mayntz (Hrsg.), Studien zum Interview. Meisenheim am Glan: Hain.

Schanz, V./Schmidt, P., 1981 : Interviewereffekte im Nationalen Sozialen Survey. Uberarbeitete (dritte) Fassung eines Referates im Rahmen einer Arbeitstagung im NSS. Mannheim. Erscheint in: K.U. Mayer/ P. Schmidt (Hrsg.), Allgemeine Bevölkerungsumfrage der Sozialwissenschaften - Beiträge zu methodischen Problemen des Allbus 1980. Frankfurt: Campus.

Scheuch, E.K., 1973: Das Interview in der Sozialforschung. S. 66-190 in: R. König (Hrsg.), Handbuch der empirischen Sozialforschung. Band 2, Stuttgart: Enke.

Sudman, S./Bradburn, N.M., 1974: Response Effects in Surveys. Chicago: Aldine.

Weede, E., 1977: Hypothesen, Gleichungen und Daten. Kronberg: Athenäum. 